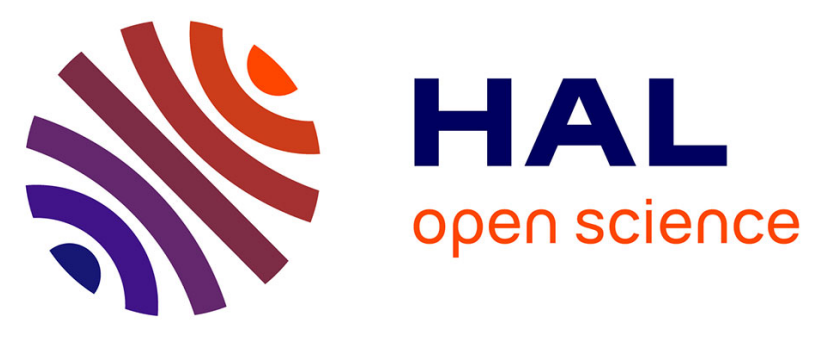

\title{
Octorotor UAVs for radar applications: modeling and analysis for control design.
}

\author{
Maria Makarov, Cristina Stoica Maniu, Sihem Tebbani, Israel Hinostroza, \\ Moreira Matheus Beltrami, Jonas Raphael Kienitz, Rafael Menegazzi, Carlos \\ Salé Moreno, Thibaut Rocheron, Joan Rojas Lombarte
}

\section{To cite this version:}

Maria Makarov, Cristina Stoica Maniu, Sihem Tebbani, Israel Hinostroza, Moreira Matheus Beltrami, et al.. Octorotor UAVs for radar applications: modeling and analysis for control design.. 2015 Workshop on Research, Education and Development of Unmanned Aerial Systems (RED-UAS 2015), Nov 2015, Cancun, Mexico. 10.1109/red-uas.2015.7441019 . hal-01259178

\section{HAL Id: hal-01259178 \\ https://hal-centralesupelec.archives-ouvertes.fr/hal-01259178}

Submitted on 6 Mar 2020

HAL is a multi-disciplinary open access archive for the deposit and dissemination of scientific research documents, whether they are published or not. The documents may come from teaching and research institutions in France or abroad, or from public or private research centers.
L'archive ouverte pluridisciplinaire HAL, est destinée au dépôt et à la diffusion de documents scientifiques de niveau recherche, publiés ou non, émanant des établissements d'enseignement et de recherche français ou étrangers, des laboratoires publics ou privés. 


\title{
Octorotor UAVs for radar applications: modeling and analysis for control design
}

\author{
Maria Makarov $^{1}$, Cristina Stoica Maniu ${ }^{1}$, Sihem Tebbani ${ }^{1}$, Israel Hinostroza ${ }^{2}$, \\ Matheus Moreira Beltrami ${ }^{3}$, Jonas Raphael Kienitz ${ }^{3}$, Rafael Menegazzi ${ }^{3}$, \\ Carlos Salle Moreno ${ }^{3}$, Thibaut Rocheron ${ }^{3}$, Joan Rojas Lombarte ${ }^{3}$
}

\begin{abstract}
In the context of radar applications using small UAVs (Unmanned Aerial Vehicles), this paper focuses on the dynamic modeling and the analysis of an octorotor in view of control design. As part of a research and educational project aiming at designing a VTOL (vertical take-off and landing) flying robot with an embedded radar system, this paper presents a complete multivariable nonlinear model of the rigid body motion of a commercially available octorotor, fully parametrized considering the geometry and mass properties of the octorotor and the load. This model is completed to take into account the effects of air drag due to the friction between the drone and the air. To meet the control objectives for radar applications, a PID (Proportional Integral Derivative) controller for altitude and attitude control, then a Linear Quadratic controller for trajectory tracking are designed. The proposed control laws are validated through simulation results and exhibit effective performance.
\end{abstract}

\section{INTRODUCTION}

Traditional aerial platforms for radar applications are airplanes and satellites [1]. With the advent of moderately low-cost UAVs (quadrotos, hexarotors), the possibility to use small UAVs as platforms for radars is substantially considered [2], [3]. Small UAVs are easy to deploy providing a cost-effective way of testing airborne sensors and related techniques. The objective of the present research and educational project is to design a VTOL (vertical take-off and landing) flying robot with

\footnotetext{
${ }^{1}$ M. Makarov, C. Stoica Maniu and S. Tebbani are with L2S, CentraleSupelec - CNRS - Université Paris-Sud, France, Gifsur-Yvette F91192. E-mail: \{maria.makarov; cristina.stoica; sihem.tebbani\}@centralesupelec.fr

${ }^{2}$ I. Hinostroza is with SONDRA Lab, CentraleSupelec, Gif-surYvette, France. E-mail: israel.hinostroza@supelec.fr

${ }^{3}$ M. M. Beltrami, J. R. Kienitz, R. Menegazzi, C. Salle Moreno, T. Rocheron, J. R. Lombarte are with the Department of Automatic Control, CentraleSupelec, France, Gif-sur-Yvette F91192
}

an embedded radar system for civilian applications like crop field monitoring, rescue operations after avalanches, damage evaluation after natural disasters etc. For the efficiency of such radar missions, a critical point that will affect the collected data is the stability of the flying platform and its ability to precisely follow a prescribed trajectory.

The long term goal of this project is to build an efficient multirotor helicopter through a simultaneous and optimized design of the mechanical structure of the flying platform and of its control system. As a first step toward this goal, this paper presents the modeling, analysis and control of a commercially available octorotor (ARFMikroKopter OktoXL, Mikrokopter, HiSystems $\mathrm{GMBH})$. The characteristics of this open-source platform are compatible with the required payload, and the redundancy provided by the eight rotors increases the drone's stability. A survey on publicly available open-source projects on UAVs can be found in [4] and some elements of comparison between plateforms are presented in [5]. A comparison between different multirotors architectures in view of a model-based design is presented in [6]. Other uses of Mikrokopter platforms for research purposes are reported in [7] and [8]. To meet the requirements of radar applications, the control system must guarantee an efficient trajectory tracking, at a constant and relatively high speed, and despite possible external wind disturbances [9]. Recently, various control schemes for attitude control or trajectory tracking have been presented for quadrotors [10], as well as other redundant drone's configurations like hexarotors [11], [12], [13] or octorotors in various configurations (4Yshaped octorotor in [14], [15], coaxial octorotor in 
[16], [17], star-shaped octorotor in [18], [19]). Depending on the application, different levels of modeling are used, from classical rigid-body dynamics to detailed aerodynamic effects [20], [21]. Linear control design (PID or optimal state-feedback) is investigated in [10], [16], [18], [17], while the other previously cited works explore non linear control methods.

In the context of radar applications using the octorotor ARF-MikroKopter OktoXL, this paper focuses on the dynamic modeling and the analysis of the proposed drone in view of control design. A complete multivariable nonlinear model of the rigid body motion is derived, fully parametrized considering the geometry and mass properties of the octorotor and the load. This model is completed to take into account the effects of air drag due to the friction between the drone and the air. A simulator is build to implement this complete nonlinear model, while a simplified model is used for control design for radar applications. Finally, in order to meet the desired specifications for radar applications, two control laws are implemented: a PID controller for altitude and attitude control and a Linear Quadratic (LQ) controller for trajectory tracking. These linear control laws are selected in order to $i$ ) first evaluate the performance and robustness of simple control structures (set of SISO controllers and control allocation in case of PID control, MIMO state-feedback controller in case of LQ control), ii) serve later for comparison with other linear or nonlinear control strategies which may be envisaged in future work.

The studied octorotor and the desired control objectives in view of radar applications are first presented in Section II. Section III is dedicated to the modeling of the dynamic behavior of the octorotor, including the air drag effect. Attitude control and optimal control for trajectory tracking are detailed in Section IV, and are illustrated in Section $\mathrm{V}$ by simulation results.

Mathematical notations. Throughout the paper, the symbols $c \phi$ and $s \phi$ denote $\cos \phi$ and $\sin \phi$, respectively. The notation 1..n represents the first $n$ non zero natural numbers. The 2-norm of the vector $\mathbf{v}$ is denoted $\|\mathbf{v}\|$. The transpose of the matrix $\boldsymbol{M}$ is denoted $\boldsymbol{M}^{T}$. The pseudo-inverse of the matrix $\boldsymbol{M}$ is denoted $\boldsymbol{M}^{+}=\left(\boldsymbol{M}^{T} \boldsymbol{M}\right)^{-1} \boldsymbol{M}^{T}$.

\section{SYSTEM DESCRIPTION AND CONTROL SPECIFICATIONS}

In this section, the specific requirements due to the envisaged radar applications are exposed and translated in control objectives. The experimental platform, the octorotor ARF-MikroKopter OktoXL [22], is then briefly presented.

\section{A. Requirements for radar applications}

Synthetic Aperture Radar (SAR) image formation is based on the coherent sum of the scattered waves due to the periodic illumination of a scene by the radar [23]. For stripmap mode, sideway illumination (relative to the UAV movement) of the scene with constant inclination, altitude, speed and linear trajectory, is desired. The radar specifications for the $\mathrm{X}$ band FM Continuous Wave Synthetic Aperture Radar to be used are summarized in Table I. Deviations from the ideal known linear trajectory induce changes in the expected phase history of the scatterers (objects in the scene), producing errors in the synthesis of the SAR image. These errors can be compensated with signal processing autofocus techniques, in combination with the information of the sensors of the UAV, up to a certain degree [24]. In order to comply with the radar specifications, by limiting the phase history errors due to trajectory deviations, the desired control performances of the UAV's movement are specified in Table I.

\section{B. Experimental platform}

The studied multirotor (Fig. 1) is equipped with eight brushless motors with twin-bladed propellers,

TABLE I: Radar and control specifications.

\begin{tabular}{ll}
\hline \multicolumn{2}{c}{ Radar specifications } \\
\hline Platform speed & $10 \mathrm{~m} / \mathrm{s}$ \\
Ground resolution & $0.8 \times 1 \mathrm{~m}$ \\
Height of flight & $100 \mathrm{~m}$ \\
Ground range & $250-550 \mathrm{~m}$ \\
\hline \multicolumn{1}{c}{ Control specifications } \\
\hline Translation speed & $10 \mathrm{~m} / \mathrm{s} \pm 0.2 \mathrm{~m} / \mathrm{s}$ \\
Maximum trajectory deviation & less than $1 \mathrm{~m}$ \\
Operational altitude & $100 \mathrm{~m}$ \\
Stabilized attitude & \\
\hline
\end{tabular}


arranged in a star-shaped way. The motors are attached to the central block with arms of different and alternating length ( $L$ for longer arms, $l$ for shorter arms). Longer arms correspond to oddnumbered motors which rotate in the clockwise direction, whereas shorter arms correspond to evennumbered motors which rotate in the counterclockwise direction. The motors placed on the longer arms have the advantage of providing higher torques because of the longer distance from the vertical axis. On the other hand, the motors placed on the short arms generate less momentum. Thus, this solution is a trade-off between the two configurations (i.e. only four long arms or only four short arms) and improves the handling ability of the drone. Choosing opposite directions of rotation between the odd- and even-numbered motors is the solution to compensate the drag torque. The motors are fixed with a $3^{\circ}$ inclination with respect to the vertical axis $\mathrm{Z}$. The sign of the angle depends on the direction of rotation of the motor as indicated in Fig. 2. The main dimensions and masses of the drone are provided in Table II (see Appendix VII-B).

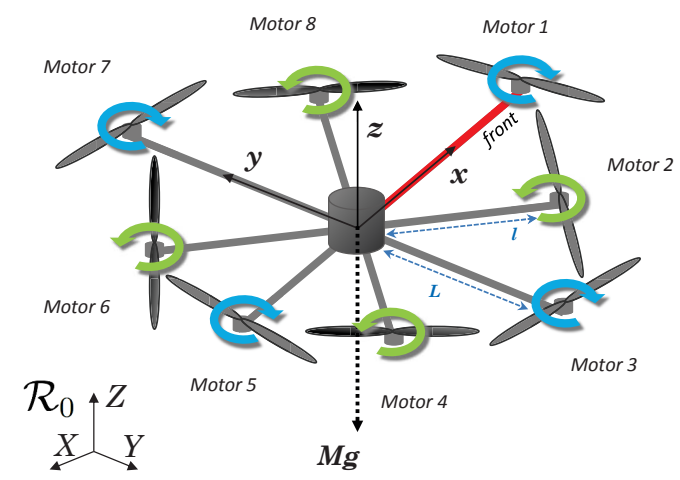

Fig. 1: Schematic of the considered octorotor and reference frames.

\section{OCTOROTOR DYNAMIC MODELING AND ANALYSIS}

The dynamic modeling of a multirotor includes the description of the rigid body dynamics in space, the aerodynamic effects, the propellers rotation, and the motor model. In this section, the rigid body dynamic equations of motion are first provided, then the air drag effect is included. Other aerodynamic effects and the model of the brushless

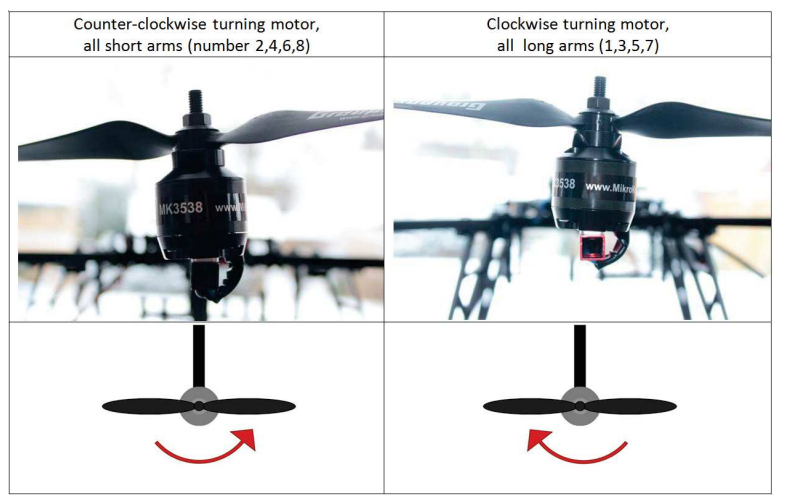

Fig. 2: Inclination of the rotor axes with respect to $Z$.

motors with their controller will be considered in future work.

\section{A. Notations and assumptions}

To derive the rigid body dynamic equations, the inertial frame $\mathcal{R}_{0}(X, Y, Z)$ and the body fixed frame $\mathcal{R}(x, y, z)$ are defined in Fig. 1 and 3.

The orientations are described using the Euler angles convention with $\varphi$ the roll angle, $\theta$ the pitch angle, $\psi$ the yaw angle. The nonlinear equations of motion in the state-space form are written using the following state vector:

$$
\boldsymbol{X}=\left(\begin{array}{llllllllllll}
X & Y & Z & \varphi & \theta & \psi & V_{x} & V_{y} & V_{z} & \omega_{x} & \omega_{y} & \omega_{z}
\end{array}\right)^{T}
$$

where $X, Y, Z$ are the position of the drone in the Earth's frame, and $V_{x}, V_{y}, V_{z}$ their time derivatives, $\varphi, \theta, \psi$ the Euler angles defining the orientation of the drone in the Earth's frame, and $\omega_{x}, \omega_{y}, \omega_{z}$ the rotational speeds of the drone in its own frame. The origin of the body fixed frame is placed in the

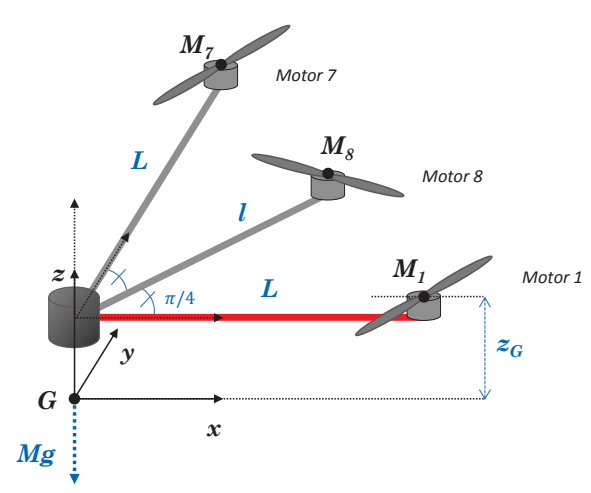

Fig. 3: Dimensions and notations - detailed view. 
center of mass $G$ of the octorotor with its payload (Fig. 3).

The rotation matrix $\boldsymbol{R}$ from the inertial frame $\mathcal{R}_{0}$ to the drone's frame $\mathcal{R}$ is defined as:

$$
\boldsymbol{R}=\left[\begin{array}{ccc}
c \psi c \theta & c \psi s \theta s \varphi-s \psi c \varphi & c \psi s \theta c \varphi+s \psi s \varphi \\
s \psi c \theta & s \psi s \theta s \varphi+c \psi c \varphi & s \psi s \theta c \varphi-c \psi s \varphi \\
-s \theta & c \theta s \varphi & c \theta c \varphi
\end{array}\right]
$$

The angular speed $\boldsymbol{\omega}^{R}=\left(\omega_{x} \omega_{y} \omega_{z}\right)^{T}$ of the drone around its axis expressed in its own frame is related to the derivatives of the Euler angles $\Theta^{R_{0}}=$ $(\varphi \theta \psi)^{T}$ by:

$$
\begin{aligned}
& \omega_{x}=\dot{\varphi}-\dot{\psi} s \theta \\
& \omega_{y}=\dot{\theta} c \varphi+\dot{\psi} s \varphi c \theta \\
& \omega_{z}=-\dot{\theta} s \varphi+\dot{\psi} c \varphi c \theta
\end{aligned}
$$

To model the rigid body motion of the drone, several assumptions are made:

A1 The structure of the drone and the propellers are rigid.

A2 The center of the body fixed frame $\mathcal{R}$ is always concurrent with the center of gravity $G$ of the drone (and thus depends on the load).

A3 The drone's inertia is symmetrical, and its center of gravity lies on the $\mathrm{Z}$ axis.

A4 The thrust force $(T)$ and drag torque $(D)$ generated by the $i$-th propeller are assumed to be proportional to the squared propeller's speed $\Omega_{i}$

$$
T_{i}=b \Omega_{i}^{2}, \quad D_{i}=d \Omega_{i}^{2} \text { for } i=1 . .8
$$

where $b$ and $d$ are respectively the thrust and drag coefficients.

A5 The brushless motor model is not added at this stage, and the considered control inputs to the system are the rotors rotational speeds $\Omega_{i, i=1 . .8}$. Taking into consideration that the studied octorotor has its motors fixed with a small inclination $\alpha$ of 3 degrees with respect to the vertical axis, the thrust and drag contributions are decomposed in vertical $\left(T_{V_{i}}, D_{V_{i}}\right)$ and horizontal components $\left(T_{H_{i}}, D_{H_{i}}\right)$ :

$$
T_{V_{i}}=T_{i} c \alpha, T_{H_{i}}=T_{i} s \alpha, D_{V_{i}}=D_{i} s \alpha, D_{H_{i}}=D_{i} c \alpha
$$

The efforts applied in $G$ (see Fig. 3) resulting from the propeller forces $\boldsymbol{F}_{x, y, z}^{R}$ and torques $\boldsymbol{\tau}_{x, y, z}^{R}$ on the drone can be expressed in the drone's frame by:

$$
\begin{aligned}
& F_{x}^{R}=T_{H_{3}}-T_{H_{7}}+\left(T_{H_{6}}+T_{H_{8}}-T_{H_{2}}-T_{H_{4}}\right) / \sqrt{2} \\
& F_{y}^{R}=T_{H_{1}}-T_{H_{5}}+\left(T_{H_{4}}+T_{H_{6}}-T_{H_{2}}-T_{H_{8}}\right) / \sqrt{2} \\
& F_{z}^{R}=\sum_{i=1}^{8} T_{V_{i}} \\
& \tau_{x}^{R}=D_{V_{3}}-D_{V_{7}}+\left(D_{V_{2}}+D_{V_{4}}-D_{V_{6}}-D_{V_{8}}\right) / \sqrt{2} \\
& +L\left(T_{V_{7}}-T_{V_{3}}\right)+l\left(T_{V_{6}}+T_{V_{8}}-T_{V_{2}}-T_{V_{4}}\right) / \sqrt{2} \\
& +z_{G}\left(T_{H_{5}}-T_{H_{1}}\right)+z_{G}\left(T_{H_{2}}+T_{H_{8}}-T_{H_{4}}-T_{H_{6}}\right) / \sqrt{2} \\
& \tau_{y}^{R}=D_{V_{1}}-D_{V_{5}}+\left(D_{V_{2}}+D_{V_{8}}-D_{V_{4}}-D_{V_{6}}\right) / \sqrt{2} \\
& +L\left(T_{V_{5}}-T_{V_{1}}\right)+l\left(T_{V_{4}}+T_{V_{6}}-T_{V_{2}}-T_{V_{8}}\right) / \sqrt{2} \\
& +z_{G}\left(T_{H_{3}}-T_{H_{7}}\right)+z_{G}\left(T_{H_{6}}+T_{H_{8}}-T_{H_{2}}-T_{H_{4}}\right) / \sqrt{2} \\
& \tau_{z}^{R}=D_{H_{1}}+D_{H_{3}}+D_{H_{5}}+D_{H_{7}}-D_{H_{2}}-D_{H_{4}} \\
& -D_{H_{6}}-D_{H_{8}}+L\left(T_{H_{1}}+T_{H_{3}}+T_{H_{5}}+T_{H_{7}}\right) \\
& -l\left(T_{H_{2}}+T_{H_{4}}+T_{H_{6}}+T_{H_{8}}\right)
\end{aligned}
$$

with $z_{G}$ defined as the distance along the Z-axis between the rotor plane and the center of mass of the drone, which is also the origin of the drone's reference frame.

\section{B. Nonlinear simulation model}

Based on the notations and assumptions detailed in the previous section, the complete nonlinear model describing the dynamics of the drone can be derived:

$$
\begin{aligned}
\dot{X}= & V_{x} \\
\dot{Y}= & V_{y} \\
\dot{Z}= & V_{z} \\
\dot{\varphi}= & \omega_{x}+s \varphi \frac{s \theta}{c \theta} \omega_{y}+c \varphi \frac{s \theta}{c \theta} \omega_{z} \\
\dot{\theta}= & c \varphi \omega_{y}-s \varphi \omega_{z} \\
\dot{\psi}= & \frac{s \varphi}{c \theta} \omega_{y}+\frac{c \varphi}{c \theta} \omega_{z} \\
m \dot{V}_{x}= & (c \psi c \theta) F_{x}^{R}+(c \psi s \theta s \varphi-s \psi c \varphi) F_{y}^{R} \\
& +(c \psi s \theta c \varphi+s \psi s \varphi) F_{z}^{R}+F_{x}^{a i r} \\
m \dot{V}_{y}= & (s \psi c \theta) F_{x}^{R}+(s \psi s \theta s \varphi+c \psi c \varphi) F_{y}^{R} \\
& +(s \psi s \theta c \varphi-c \psi s \varphi) F_{z}^{R}+F_{y}^{a i r} \\
m \dot{V}_{z}= & (-s \theta) F_{x}^{R}+(c \theta s \varphi) F_{y}^{R}+(c \theta c \varphi) F_{z}^{R} \\
& +F_{z}^{a i r}-m g \\
I_{x x} \dot{\omega}_{x}= & \left(I_{y y}-I_{z z}\right) \omega_{y} \omega_{z}+\tau_{x}^{R} \\
I_{y y} \dot{\omega}_{y}= & \left(I_{z z}-I_{x x}\right) \omega_{x} \omega_{z}+\tau_{y}^{R} \\
I_{z z} \dot{\omega}_{z}= & \left(I_{x x}-I_{y y}\right) \omega_{x} \omega_{y}+\tau_{z}^{R}
\end{aligned}
$$


This model has eight inputs (rotational speed of each motor $\Omega_{i}$ ) entering the equations through $F_{x}^{R}$, $F_{y}^{R}, F_{z}^{R}$ and $\tau_{x}^{R}, \tau_{y}^{R}, \tau_{z}^{R}$ detailed in the previous section. It is implemented in Matlab/Simulink for further control laws validation. The mass $m$ and the inertia components $I_{x x}, I_{y y}, I_{z z}$ of the drone are determined from the masses and the inertias of the motors, the main bloc, the battery and the load. This model takes account of the load (small radar system for example) and the number of batteries with the calculation of $z_{G}$.

Additional terms $F_{x}^{a i r}, F_{y}^{a i r}, F_{z}^{a i r}$ are included to represent the components of the air drag force $\boldsymbol{F}^{\text {air }}$ on the drone's structure. The air drag effects are assumed to be proportional to the square of the speed of the object relative to the air $\boldsymbol{V}_{\text {rel }}=$ $\left(V_{\text {rel }_{x}} V_{\text {rel }_{y}} V_{\text {rel }_{z}}\right)^{T}$ :

$$
\boldsymbol{F}^{a i r}=-d_{a i r}\left\|\boldsymbol{V}_{\text {rel }}\right\| \boldsymbol{V}_{\text {rel }}
$$

At the present point of this research and educational project, a simple model is selected based on the capacity to reproduce the resistant aspects of the air that are observed in a real case. A first simplification assumes that the resultant air drag force is applied to the drone's center of mass. From aerodynamics, $d_{\text {air }}=\rho S C_{D} / 2$ with $\rho$ the density of the air, $S$ the reference area and $C_{D}$ the drag coefficient which depends on the shape of the object and the Reynolds number. A complete model of the air drag effects requires the identification of the parameters $C_{D}$ and $S$, which requires wind tunnel tests and numerical methods. Here, the term $d_{\text {air }}$ is assumed to be constant and the same in all directions. This simplification is however legitimate in the considered operational conditions. In the following, the value of $d_{\text {air }}=$ $0.03 \mathrm{~m}^{-1} \cdot \mathrm{kg}$ is used, but further identification need to be performed to obtain a reliable model.

\section{Simplified nonlinear model for control design}

For control design, linearized models are derived in Section IV. Before this step, the nonlinear equations (20) to (25) are simplified, based on the relative contributions of $T_{H}, T_{V}, D_{H}, D_{V}$.

S1 In (20)-(22), $F_{x}^{R}$ and $F_{y}^{R}$ are negligible compared to $F_{z}^{R}$, since $T_{H_{i}}<<T_{V_{i}}$.

S2 In (11)-(12) and therefore in (23)-(24), $D_{V_{i}}$ is negligible compared to $L T_{V_{i}}$.
S3 In (13), $D_{H_{i}}$ and $L T_{H_{i}}$ or $l T_{H_{i}}$ are of the same order of magnitude.

In the following, ideal still weather condition is supposed (no wind), and a displacement along $X$ is considered, leading to $V_{r e l_{x}}=V_{x}$, and $V_{\text {rel }}=V_{y}=0$. Even if $V_{z} \neq 0$ during the takeoff phase, no air-drag contribution is considered in the $Z$ direction. These simplifications result in the following simplified nonlinear model, in which the neglected terms will be treated as disturbances:

$$
\begin{aligned}
m \dot{V}_{x}= & (c \psi s \theta c \varphi+s \psi s \varphi) \sum_{i=1}^{8} T_{V_{i}}-d_{a i r} V_{x}^{2} \\
m \dot{V}_{y}= & (s \psi s \theta c \varphi-c \psi s \varphi) \sum_{i=1}^{8} T_{V_{i}} \\
m \dot{V}_{z}= & (c \theta c \varphi) \sum_{i=1}^{8} T_{V_{i}}-m g \\
I_{x x} \dot{\omega}_{x}= & \left(I_{y y}-I_{z z}\right) \omega_{y} \omega_{z}+L\left(T_{V_{7}}-T_{V_{3}}\right) \\
& +l\left(T_{V_{6}}+T_{V_{8}}-T_{V_{2}}-T_{V_{4}}\right) / \sqrt{2} \\
I_{y y} \dot{\omega}_{y}= & \left(I_{z z}-I_{x x}\right) \omega_{x} \omega_{z}+L\left(T_{V_{5}}-T_{V_{1}}\right) \\
& +l\left(T_{V_{4}}+T_{V_{6}}-T_{V_{2}}-T_{V_{8}}\right) / \sqrt{2} \\
I_{z z} \dot{\omega}_{z}= & \left(I_{x x}-I_{y y}\right) \omega_{x} \omega_{y}+D_{H_{1}}+D_{H_{3}}+D_{H_{5}} \\
& +D_{H_{7}}-D_{H_{2}}-D_{H_{4}}-D_{H_{6}}-D_{H_{8}} \\
& +L\left(T_{H_{1}}+T_{H_{3}}+T_{H_{5}}+T_{H_{7}}\right) \\
& -l\left(T_{H_{2}}+T_{H_{4}}+T_{H_{6}}+T_{H_{8}}\right)
\end{aligned}
$$

\section{CONTROL DESIGN}

As part of this educational project in the context of radar applications, two control designs are proposed - a PID controller for altitude and attitude control (Section IV-B), and a LQ controller for trajectory control allowing a translational movement at a constant velocity (Section IV-C), both based on a linearized model of the octorotor presented in Section IV-A. Wind influence is not explicitly taken into account at this point, and is treated as a disturbance.

\section{A. Linearized model}

A general linearized model is computed based on the equations (14)-(19) and (27)-(32), valid at an equilibrium point or trajectory of the nonlinear system. The model is written using the state vector (1), and the following control vector:

$$
\boldsymbol{U}=\left(\begin{array}{llllllll}
\Omega_{1} & \Omega_{2} & \Omega_{3} & \Omega_{4} & \Omega_{5} & \Omega_{6} & \Omega_{7} & \Omega_{8}
\end{array}\right)^{T}
$$


The linearization point is characterized by: null or constant velocity $V_{x}$, null velocities $\bar{V}_{y}, \bar{V}_{z}$, null or constant angle $\bar{\theta}$, null angles $\bar{\varphi}, \bar{\psi}$, and null rotational velocities $\bar{\omega}_{x}, \bar{\omega}_{y}, \bar{\omega}_{z}$. The notations $\cdot$ and $\tilde{*}$ denote respectively the equilibrium value of the variables, and the small deviations from this operating point:

$$
\tilde{\boldsymbol{X}}=\boldsymbol{X}-\overline{\boldsymbol{X}}, \tilde{\Omega}_{i}=\Omega_{i}-\bar{\Omega}_{i}, \tilde{\boldsymbol{U}}=\boldsymbol{U}-\overline{\boldsymbol{U}}
$$

The linearized model can be written as follows:

$$
\dot{\tilde{X}}=A \tilde{X}+B \tilde{U}
$$

The details of the matrices are presented in the Appendix VII-A. The hovering model (stationary flight at constant height) can be obtained from this generic model by replacing $\bar{\theta}$ and $\bar{V}_{x}$ by 0 .

\section{B. Altitude and attitude control (PID)}

In this section, a decoupled PID controller is designed based on the hovering model to control separately the altitude $\tilde{Z}$ and the attitude angles $\tilde{\varphi}, \tilde{\theta}, \tilde{\psi}$. A reduced state vector $\boldsymbol{X}^{\prime}$ composed of the four considered variables and their derivatives is used. Instead of the rotor rotation speed vector (33), a fictitious input vector $\boldsymbol{U}^{\prime}$ directly related to the generated forces and torques is used:

$$
\begin{aligned}
\tilde{\boldsymbol{X}}^{\prime} & =\left(\begin{array}{llllllll}
\tilde{Z} & \tilde{\varphi} & \tilde{\theta} & \tilde{\psi} & \tilde{V}_{z} & \tilde{\omega}_{x} & \tilde{\omega}_{y} & \tilde{\omega}_{z}
\end{array}\right)^{T} \\
\tilde{U}^{\prime} & =\left(\begin{array}{lllll}
\tilde{F}_{z} & \tilde{u}_{x} & \tilde{u}_{y} & \tilde{u}_{z}
\end{array}\right)^{T}
\end{aligned}
$$

The new model can be written as follows:

$$
\dot{\tilde{X}}^{\prime}=A^{\prime} \tilde{X}^{\prime}+B^{\prime} \tilde{U}=A^{\prime} \tilde{X}^{\prime}+B^{\prime \prime} \tilde{U}^{\prime}
$$

where the matrices $\boldsymbol{A}^{\prime}$ and $\boldsymbol{B}^{\prime}$ denote the submatrices extracted from the complete model $\boldsymbol{A}$ and $\boldsymbol{B}$ provided in (49). The matrix $\boldsymbol{B}^{\prime \prime}$ denotes the new input matrix. In hovering mode, the following matrices are considered:

$$
\begin{aligned}
\boldsymbol{A}^{\prime} & =\left(\begin{array}{ll}
\mathbf{0}_{4} & \boldsymbol{I}_{4} \\
\mathbf{0}_{4} & \mathbf{0}_{4}
\end{array}\right) \\
\boldsymbol{B}^{\prime \prime} & =\left(\begin{array}{c}
\mathbf{0}_{8 \times 4} \\
\boldsymbol{B}_{2}^{\prime \prime}
\end{array}\right), \boldsymbol{B}_{2}^{\prime \prime}=\operatorname{diag}\left(\frac{1}{m}, \frac{1}{I_{x x}}, \frac{1}{I_{y y}}, \frac{1}{I_{z z}}\right)
\end{aligned}
$$

The obtained model is a set of four decoupled double integrator systems. In the Laplace domain, with $\square(s)$ denoting the Laplace transform of $\square(t)$, the input-output transfer functions are:

$$
\begin{aligned}
& Z(s)=\frac{1}{m s^{2}} F_{z}(s), \quad \varphi(s)=\frac{1}{I_{x x} s^{2}} \tau_{x}(s), \\
& \theta(s)=\frac{1}{I_{y y} s^{2}} \tau_{y}(s), \quad \psi(s)=\frac{1}{I_{z z} s^{2}} \tau_{z}(s)
\end{aligned}
$$

Four different SISO (Single-Input SingleOutput) PID controllers are designed for altitude, pitch, roll and yaw control:

$$
C_{P I D_{i}}(s)=K_{P_{i}}\left(1+\frac{1}{T_{I_{i}} s}+\frac{T_{D_{i}} s}{1+\tau_{D_{i}} s}\right), \quad i=1 . .4
$$

The classical tuning parameters are $K_{P_{i}}$ (the proportional term), $T_{I_{i}}$ (the integral term) and $T_{D_{i}}$ (the derivative term filtered with a smaller time constant $\left.\tau_{D_{i}}=T_{D_{i}} / 10\right)$. All the four controllers are tuned to achieve similar dynamics in the four considered variables, with about $55^{\circ}$ of phase margin, 10rad/s open-loop cut-off frequency resulting in $28 \%$ overshoot at about $0.3 \mathrm{~s}$. Therefore, only the gain $K_{P_{i}}$ varies between the four controllers, depending of the inertia or mass values of the different systems.

The actual input vector $U$ can be computed from the fictitious one $U^{\prime}$ as follows:

$$
B^{\prime} \tilde{U}=B^{\prime \prime} \tilde{U}^{\prime} \Rightarrow \tilde{U}=\underbrace{\left(B^{\prime}\right)^{+} B^{\prime \prime}}_{M_{u}} \tilde{U}^{\prime}
$$

where $(\cdot)^{+}$denotes the pseudo-inverse of the matrix $\boldsymbol{B}^{\prime}$. Other solutions for control allocation may be found in [25]. As discussed in [26] and illustrated in [27], [19], such a modular control design with a separate control allocator provides some benefits in case of actuator constraints. The resulting control scheme appears in Fig. 4.

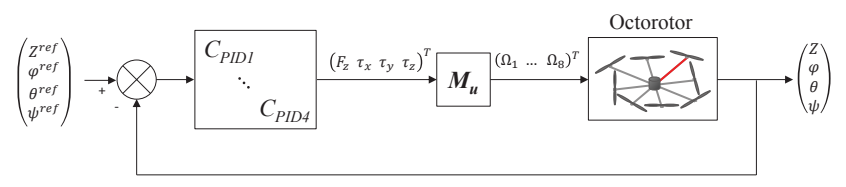

Fig. 4: PID for altitude and attitude control

\section{Trajectory tracking control $(L Q)$}

Trajectory tracking control can be achieved i) either by completing an attitude and altitude controller by an outer loop providing the necessary angle references, ii) or by directly designing a MIMO controller based on the complete linearized model which controls the altitude, the attitude and the position at the same time. For the complete linearized model (35), the LQ (Linear Quadratic) method was selected. Indeed, it can easily deal with MIMO (Multi Input Multi Output) systems, 
provides a good level of robustness with respect to model uncertainties (several parameters in the model such as the drag and lift coefficients are not precisely known), and is tuned via an optimization method, assuring optimal stability margins.

LQ state feedback control supposes that the full state vector is accessible. On the considered octorotor, three gyroscopes, three accelerometers and a magnetometer are available for attitude estimation and provide the angular velocities in the drone's frame. An altimeter and the GPS make possible the estimation of the positions and translational velocities. The design of a detailed procedure for the state estimation is left for future work, and the necessary measurements of the state vector are assumed to be available in what follows.

The considered control scheme is represented in Fig. 5, where $\boldsymbol{K}_{L Q}$ is the feedback gain matrix, and $\boldsymbol{K}_{\text {ref }}$ the reference gain matrix. Optimal state feedback $\tilde{\boldsymbol{U}}=\boldsymbol{K}_{\text {ref }} \tilde{\boldsymbol{X}}_{\boldsymbol{r}} \boldsymbol{e} \boldsymbol{f}-\boldsymbol{K}_{L Q} \tilde{\boldsymbol{X}}$ is classically computed to minimize the cost:

$$
\begin{aligned}
J & =\int_{0}^{+\infty}\left(\tilde{\boldsymbol{X}}^{T} \boldsymbol{Q} \tilde{\boldsymbol{X}}+\tilde{\boldsymbol{U}}^{T} \boldsymbol{R} \tilde{\boldsymbol{U}}\right) d t \\
\boldsymbol{Q} & =\operatorname{diag}\left(q_{i, i=1 . .12}\right), \quad \boldsymbol{R}=\boldsymbol{I}_{\mathbf{8}}
\end{aligned}
$$

The weightings on the state $Q$ are selected to emphasize the trajectory control objectives, i.e. the variables $X, Y, \theta$ a,d $V_{x}: q_{1,2,5}=10000, q_{3}=$ $1000, q_{4,6.12}=1$. The matrix $\boldsymbol{K}_{\text {ref }}=-((\boldsymbol{A}-$ $\left.\left.\boldsymbol{B} \boldsymbol{K}_{L Q}\right)^{-1} \boldsymbol{B}\right)^{+}$ensures a zero static error with respect to the reference. To cancel the static error in presence of disturbances, a Linear Quadratic Integral (LQI) controller can be considered in future work.

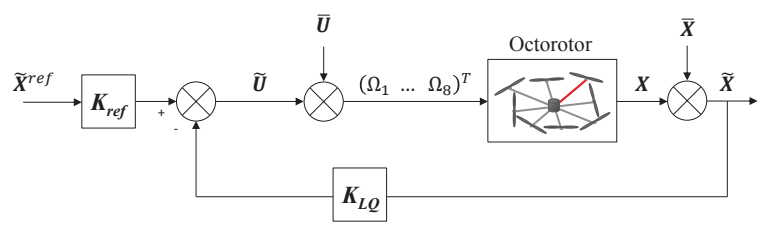

Fig. 5: Linear quadratic control for trajectory tracking

\section{Simulation Results}

The previously designed control laws are applied to the complete nonlinear model. PID control is applied for the take-off (0-20s), then
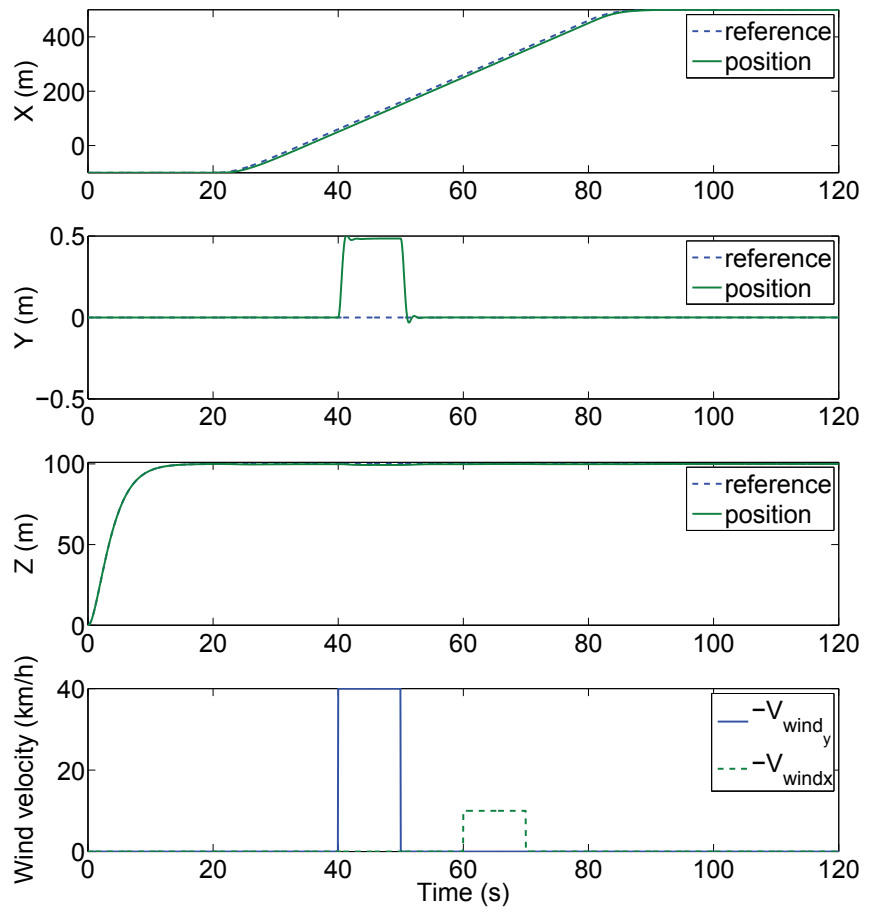

Fig. 6: Positions $X, Y, Z$ and wind disturbance profile
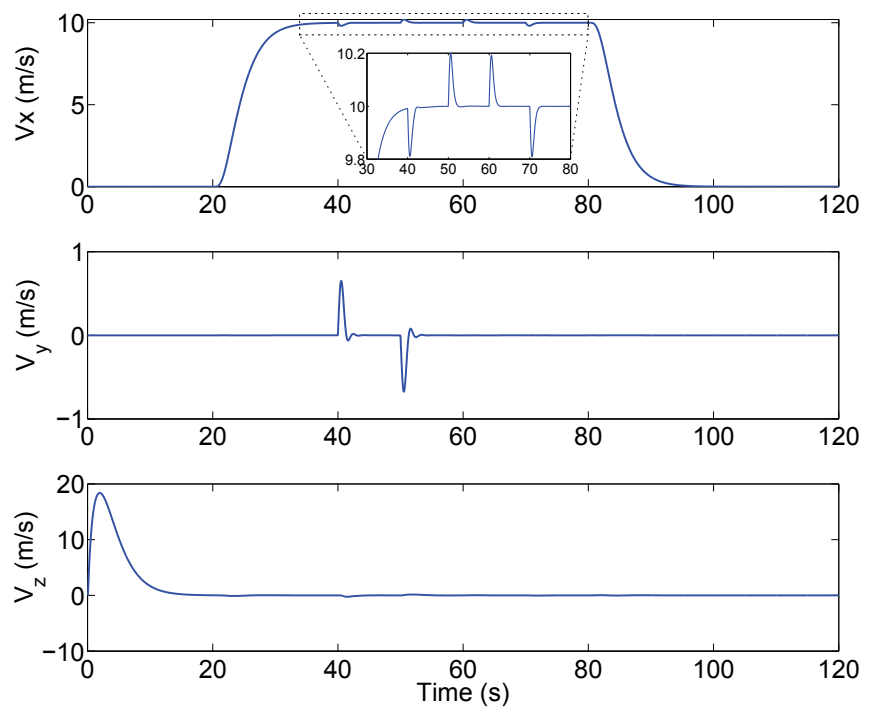

Fig. 7: Speeds $V_{x}, V_{y}, V_{z}$

the trajectory tracking and disturbance rejection is assured by the LQ controller (20-120s). Step disturbances representing wind gusts of $40 \mathrm{~km} / \mathrm{h}$ in the $-Y$ direction (from 40 to $50 \mathrm{~s}$ ), and $10 \mathrm{~km} / \mathrm{h}$ in the $-X$ direction (from 60 to 70 s) are applied (Fig. 6).

The performed simulations on the complete nonlinear model validate the proposed design with respect to the radar specifications. A constant 

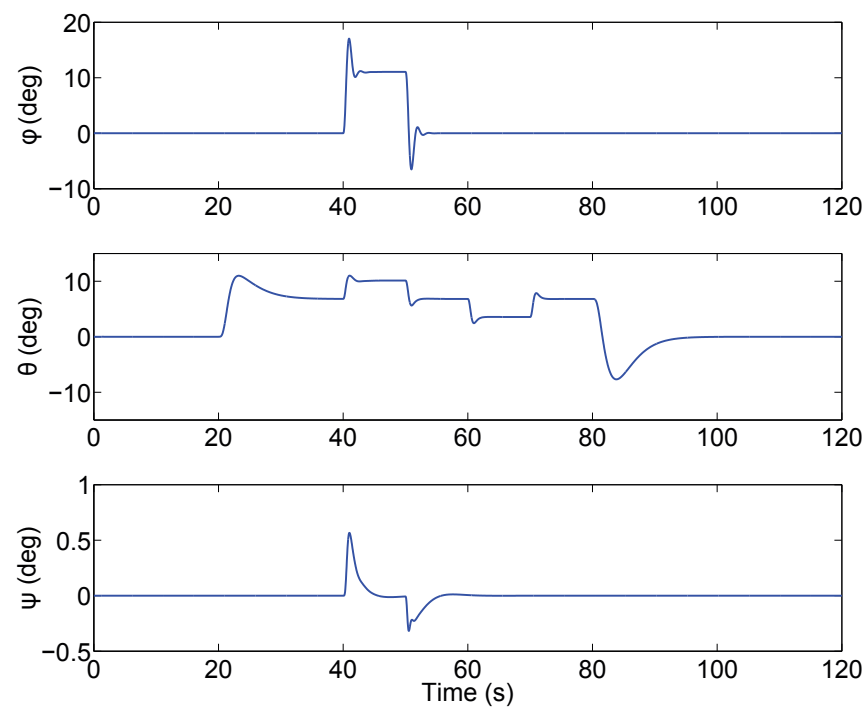

Fig. 8: Angles $\varphi, \theta, \psi$

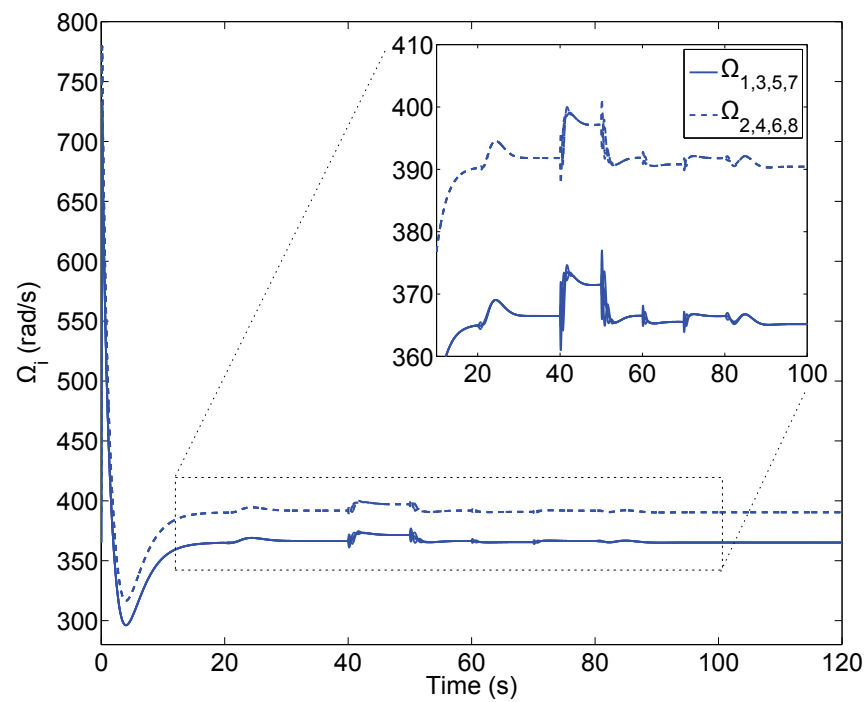

Fig. 9: Control inputs - motor speeds $\Omega_{i}$ (odd numbered - solid line, even numbered - dotted line)

linear speed is achieved along $X$ with an error no bigger than $2 \mathrm{~cm} / \mathrm{s}$ in presence of the tested wind disturbances (Fig. 7), and position deviation from the imposed trajectory is smaller than $1 \mathrm{~m}$ on $Y$ and $Z$ (Fig. 6). Attitude angles are represented in Fig. 8. Control signals are of reasonable amplitude (Fig. 9).

\section{CONCLUSION}

This paper presented the modeling of an octorotor for radar applications. A nonlinear model of the rigid body motion and air drag effect has been de- veloped and used to build a Matlab/Simulink simulator. This mathematical model of an existing UAV platform serves as the starting point of the study, and will be used to jointly optimize the mechanical and control design for radar applications. Preliminary control design based on Proportional Integral Derivative and Linear Quadratic controllers fulfills the requirements related to the envisaged Synthetic Aperture Radar image acquisitions.

In future work, further modeling effort will be dedicated to the modeling of the motor dynamics and the identification of air drag model. The design of a state estimator taking into account the availability of the measurements at different sampling times will be studied, aiming to an experimental evaluation of the proposed control laws. Particular attention will be dedicated to the energy efficiency of the designed control laws in order to optimize the battery use and thus the flight time.

\section{APPENDIX}

\section{A. Linearized model matrices}

The matrices in the linearized model can be written as:

$$
\begin{aligned}
\boldsymbol{A} & =\left(\begin{array}{cccc}
\mathbf{0}_{3} & \mathbf{0}_{3} & \boldsymbol{I}_{3} & \mathbf{0}_{3} \\
\mathbf{0}_{3} & \mathbf{0}_{3} & \mathbf{0}_{3} & \boldsymbol{A}_{24} \\
\mathbf{0}_{3} & \boldsymbol{A}_{32} & \boldsymbol{A}_{33} & \mathbf{0}_{3} \\
\mathbf{0}_{3} & \mathbf{0}_{3} & \mathbf{0}_{3} & \mathbf{0}_{3}
\end{array}\right), \boldsymbol{B}=\left(\begin{array}{c}
\mathbf{0}_{6 \times 8} \\
\boldsymbol{B}_{1} \\
\boldsymbol{B}_{2}
\end{array}\right) \\
\boldsymbol{A}_{24} & =\left(\begin{array}{ccc}
1 & 0 & \tan \bar{\theta} \\
0 & 1 & 0 \\
0 & 0 & \frac{1}{c \bar{\theta}}
\end{array}\right) \boldsymbol{A}_{32}=\left(\begin{array}{ccc}
0 & g c \bar{\theta} & 0 \\
-g & 0 & g s \bar{\theta} \\
0 & -g s \bar{\theta} & 0
\end{array}\right)(50) \\
\boldsymbol{A}_{33} & =\left(\begin{array}{ccc}
-2 d_{\text {air }} \bar{V}_{x} & 0 & 0 \\
0 & 0 & 0 \\
0 & 0 & 0
\end{array}\right)
\end{aligned}
$$

where $\boldsymbol{B}_{1}$ and $\boldsymbol{B}_{2}$ are detailed in (47), $g$ is the gravity acceleration, (48) with $p_{1}=d c \alpha+b l s \alpha$ and $p_{2}=d c \alpha+b L s \alpha$.

In (47)-(48), the equilibrium values of the rotor speeds $\bar{\Omega}_{i}$ correspond to a stationary flight at a constant altitude (hovering without wind, with no yaw torque). In these conditions, the nonlinear equations (14)-(19) and (27)-(32) reduce to:

$$
\begin{aligned}
m g & =F_{z}^{R} \\
0 & =\tau_{z}^{R}
\end{aligned}
$$

Denoting $k$ the factor between the rotation speeds such that $\Omega_{o d d}=k \Omega_{e v e n}$ with $\Omega_{o d d} \widehat{=} \Omega_{i, i=1,3,5,7}$ 


$$
\begin{aligned}
\boldsymbol{B}_{1}=\left(\begin{array}{cccccccc}
\frac{2 b c \alpha}{m} s \bar{\theta} \bar{\Omega}_{1} & \frac{2 b c \alpha}{m} s \bar{\theta} \bar{\Omega}_{2} & \frac{2 b c \alpha}{m} s \bar{\theta} \bar{\Omega}_{3} & \frac{2 b c \alpha}{m} s \bar{\theta} \bar{\Omega}_{4} & \frac{2 b c \alpha}{m} s \bar{\theta} \bar{\Omega}_{5} & \frac{2 b c \alpha}{m} s \bar{\theta} \bar{\Omega}_{6} & \frac{2 b c \alpha}{m} s \bar{\theta} \bar{\Omega}_{7} & \frac{2 b c \alpha}{m} s \bar{\theta} \bar{\Omega}_{8} \\
0 & 0 & 0 & 0 & 0 & 0 & 0 & 0 \\
\frac{2 b c \alpha}{m} c \bar{\theta} \bar{\Omega}_{1} & \frac{2 b c \alpha}{m} c \bar{\theta} \bar{\Omega}_{2} & \frac{2 b c \alpha}{m} c \bar{\theta} \bar{\Omega}_{3} & \frac{2 b c \alpha}{m} c \bar{\theta} \bar{\Omega}_{4} & \frac{2 b c \alpha}{m} c \bar{\theta} \bar{\Omega}_{5} & \frac{2 b c \alpha}{m} c \bar{\theta} \bar{\Omega}_{6} & \frac{2 b c \alpha}{m} c \bar{\theta} \bar{\Omega}_{7} & \frac{2 b c \alpha}{m} c \bar{\theta} \overline{\Omega_{8}}
\end{array}\right) \\
\boldsymbol{B}_{2}=\left(\begin{array}{ccccccccc}
0 & -\frac{2 b c \alpha}{I_{x x}} \frac{l}{\sqrt{2}} \bar{\Omega}_{2} & -\frac{2 b c \alpha}{I_{x x}} L \bar{\Omega}_{3} & -\frac{2 b c \alpha}{I_{x x}} \frac{l}{\sqrt{2}} \bar{\Omega}_{4} & 0 & \frac{2 b c \alpha}{I_{x x}} \frac{l}{\sqrt{2}} \bar{\Omega}_{6} & \frac{2 b c \alpha}{I_{x x}} L \bar{\Omega}_{7} & \frac{2 b c \alpha}{I_{x x}} \frac{l}{\sqrt{2}} \bar{\Omega}_{8} \\
-\frac{2 b c \alpha}{I_{y y}} L \bar{\Omega}_{1} & -\frac{2 b c \alpha}{I_{y y}} \frac{l}{\sqrt{2}} \bar{\Omega}_{2} & 0 & \frac{2 b c \alpha}{I_{y y}} \frac{l}{\sqrt{2}} \bar{\Omega}_{4} & \frac{2 b c \alpha}{I_{y y}} L \bar{\Omega}_{5} & \frac{2 b c \alpha}{I_{y y}} \frac{l}{\sqrt{2}} \bar{\Omega}_{6} & 0 & -\frac{2 b c \alpha}{I_{y y}} \frac{l}{\sqrt{2}} \bar{\Omega}_{8} \\
\frac{2}{I_{z z}} p_{2} \bar{\Omega}_{1} & -\frac{2}{I_{z z}} p_{1} \bar{\Omega}_{2} & \frac{2}{I_{z z}} p_{2} \bar{\Omega}_{3} & -\frac{2}{I_{z z}} p_{1} \bar{\Omega}_{4} & \frac{2}{I_{z z}} p_{2} \bar{\Omega}_{5} & -\frac{2}{I_{z z}} p_{1} \bar{\Omega}_{6} & \frac{2}{I_{z z}} p_{2} \bar{\Omega}_{7} & -\frac{2}{I_{z z}} p_{1} \bar{\Omega}_{8}
\end{array}\right)
\end{aligned}
$$

and $\Omega_{\text {even }} \widehat{=} \Omega_{i, i=2,4,6,8}$, the previous equations become:

$$
\begin{aligned}
m g & =4\left(1+k^{2}\right) b c \alpha \Omega_{\text {even }} \\
0 & =\left(4\left(k^{2}-1\right) d c \alpha+4 b s \alpha\left(L k^{2}-l\right)\right) \Omega_{\text {even }}
\end{aligned}
$$

Finally, the rotor speeds at equilibrium $\bar{\Omega}_{i}$ in stationary flight are computed using the following relationships:

$$
\begin{aligned}
k & =\frac{\Omega_{\text {odd }}}{\Omega_{\text {even }}}=\sqrt{\frac{d c \alpha+b l s \alpha}{d c \alpha+b L s \alpha}} \\
\Omega_{\text {even }}^{2} & =\frac{m g}{4\left(1+k^{2}\right) b c \alpha}
\end{aligned}
$$

\section{B. Numerical values}

The numerical values used in this study are summarized in Table II.

TABLE II: Dimensions and masses.

\begin{tabular}{ll}
\hline Parameter & Notation and value (units) \\
\hline Mass of 1 motor & $m_{m}=0.1(\mathrm{~kg})$ \\
Mass of 1 battery & $m_{b}=0.534(\mathrm{~kg})$ \\
$\begin{array}{l}\text { Mass of central block } \\
\text { Total mass with 1 battery }\end{array}$ & $m_{c}=1.226(\mathrm{~kg})$ \\
$\begin{array}{l}\text { Motor dimensions } \\
\text { (radius, height) }\end{array}$ & $r_{m}=0.56(\mathrm{~kg})$ \\
$\begin{array}{l}\text { Central block dimensions } \\
\text { (radius, height) }\end{array}$ & $r_{c}=0.09, h_{c}=0.095(\mathrm{~m})$ \\
$\begin{array}{l}\text { Battery dimensions } \\
\text { (width, length, height) }\end{array}$ & $w_{c}=0.132$, \\
Arm lengths (short, long) & $l_{c}=0.09, h_{c}=0.02(\mathrm{~m})$ \\
& $L=0.455, l=0.349(\mathrm{~m})$ \\
\hline
\end{tabular}

\section{REFERENCES}

[1] A. Moreira, P. Prats-Iraola, M. Younis, G. Krieger, I. Hajnsek, and K. P. Papathanassiou, "A tutorial on synthetic aperture radar," IEEE Geoscience and Remote Sensing Magazine, vol. 1, no. 1, pp. 6-43, 2013.
[2] E. C. Zaugg, D. L. Hudson, and D. G. Long, "The BYU SAR: A small, student-built SAR for UAV operation," in IEEE Int. Conf. on Geoscience and Remote Sensing Symposium, 2006, pp. 411-414.

[3] J.-T. González-Partida, P. Almorox-González, M. BurgosGarcía, and B.-P. Dorta-Naranjo, "SAR system for UAV operation with motion error compensation beyond the resolution cell," Sensors, vol. 8, no. 5, pp. 3384-3405, 2008.

[4] H. Lim, J. Park, D. Lee, and H. J. Kim, "Build your own quadrotor: Open-source projects on unmanned aerial vehicles," IEEE Robotics \& Automation Magazine, vol. 19, no. 3, pp. 33-45, 2012.

[5] J. Moyano Cano, "Quadrotor UAV for wind profile characterization," Master's thesis, 2013.

[6] C. Månsson and D. Stenberg, "Model-based design development and control of a wind resistant multirotor UAV," Master's thesis, Lund University, Automatic Control, 2014.

[7] I. Sa and P. Corke, "System identification, estimation and control for a cost effective open-source quadcopter," in IEEE Int. Conf. on Robotics and Automation (ICRA), 2012, pp. 2202-2209.

[8] K. Bergman and J. Ekström, "Modeling, estimation and attitude control of an octorotor using PID and L1 adaptive control techniques," Master's thesis, Linköping University, Automatic Control, The Institute of Technology, 2014.

[9] I. Sarras and H. Siguerdidjane, "On the guidance of a UAV under unknown wind disturbances," in IEEE Conf. on Control Applications (CCA), 2014, pp. 820-825.

[10] S. Bouabdallah, A. Noth, and R. Siegwart, "PID vs LQ control techniques applied to an indoor micro quadrotor," in IEEE/RSJ Int. Conf. on Intelligent Robots and Systems (IROS), vol. 3, 2004, pp. 2451-2456.

[11] T. Magnusson, "Attitude control of a hexarotor," Master's thesis, Linköping University, Automatic Control, The Institute of Technology, 2014.

[12] M. W. Achtelik, S. Lynen, M. Chli, and R. Siegwart, "Inversion based direct position control and trajectory following for micro aerial vehicles," in IEEE/RSJ Int. Conf. on Intelligent Robots and Systems (IROS), 2013, pp. 2933-2939.

[13] S. Omari, M.-D. Hua, G. Ducard, and T. Hamel, "Hardware and software architecture for nonlinear control of multirotor helicopters," IEEE/ASME Transactions on Mechatronics, vol. 18, no. 6, pp. 1724-1736, 2013.

[14] V. G. Adir, A. M. Stoica, and J. F. Whidborne, "Sliding mode control of a 4Y octorotor," UPB Sci. Bull., Series D, vol. 74, no. 4, pp. 37-51, 2012.

[15] I. Khabbazi and R. Ghasemi, "Designing back-stepping sliding mode controller for a class of 4 y octorotor," Int. Journal 
of Mechanical, Aerospace, Industrial and Mechatronics Engineering, vol. 8, no. 11, pp. 1852 - 1857, 2014.

[16] S. J. Haddadi and P. Zarafshan, "Attitude control of an autonomous octorotor," in RSI/ISM Int. Conf. on Robotics and Mechatronics (ICRoM). IEEE, 2014, pp. 540-545.

[17] F. Rinaldi, A. Gargioli, and F. Quagliotti, "PID and LQ Regulation of a Multirotor Attitude: Mathematical Modelling, Simulations and Experimental Results," Journal of Intelligent and Robotic Systems, vol. 73, no. 1-4, pp. 33-50, 2014.

[18] V. G. Adir and A. M. Stoica, "Integral LQR control of a star-shaped octorotor," INCAS Bulletin, vol. 4, no. 2, 2012.

[19] H. Alwi and C. Edwards, "Fault tolerant control of an octorotor using LPV based sliding mode control allocation," in IEEE American Control Conf. (ACC), 2013, pp. 65056510.

[20] R. Mahony, V. Kumar, and P. Corke, "Multirotor aerial vehicles: Modeling, estimation, and control of quadrotor," IEEE Robotics \& Automation Magazine, no. 19, pp. 20-32, 2012.

[21] G. M. Hoffmann, H. Huang, S. L. Waslander, and C. J. Tomlin, "Precision flight control for a multi-vehicle quadrotor helicopter testbed," Control engineering practice, vol. 19, no. 9, pp. 1023-1036, 2011.

[22] HiSystems GmbH, "ARF-MikroKopter OktoXL Manual," 2014.

[23] S. Kingsley and Q. S., Understanding Radar Systems. UK: McGraw-Hill, 1992.

[24] W. G. Carrara, R. S. Goodman, and R. M. Majewski, Spotlight Synthetic Aperture Radar: Signal Processing Algorithms. MA., USA: Artech House, 1995.

[25] G. Ducard and M.-D. Hua, "Discussion and practical aspects on control allocation for a multi-rotor helicopter," in Conf. on Unmanned Aerial Vehicle in Geomatics, 2011, pp. 1-6.

[26] O. Härkegård and S. T. Glad, "Resolving actuator redundancy - optimal control vs. control allocation," Automatica, vol. 41, no. 1, pp. 137-144, 2005.

[27] A. Marks, J. Whidborne, and I. Yamamoto, "Control allocation for fault tolerant control of a VTOL octorotor," in UKACC Int. Conf. on Control, 2012, pp. 357-362. 